Chronic Obstructive Pulmonary Diseases:

Journal of the COPD Foundation

\author{
Original Research
}

\title{
The COPD Biomarkers Qualification Consortium Database: Baseline Characteristics of the St George's Respiratory Questionnaire Dataset
}

\author{
Maggie Tabberer, $\mathrm{MSc}^{1}$ Victoria S. Benson, $\mathrm{PhD}^{1}$ Heather Gelhorn, $\mathrm{PhD}^{2}$ Hilary Wilson, $\mathrm{PhD}^{2}$ \\ Niklas Karlsson, $\mathrm{PhD}^{3}$ Hana Müllerova, $\mathrm{PhD}^{1}$ Shailendra Menjoge, $\mathrm{PhD}^{4}$ Stephen I. Rennard, $\mathrm{MD}^{5,6}$ \\ Ruth Tal-Singer, $\mathrm{PhD}^{7}$ Debora Merrill, $\mathrm{MBA}^{8}$ Paul W. Jones, PhD, FRCP, FERS ${ }^{1,9}$ for the COPD Biomarkers \\ Qualification Consortium
}

\section{Abstract}

The COPD Biomarkers Qualification Consortium (CBQC) is a public-private partnership formed in 2010 with a goal of qualifying biomarkers and clinical assessment tools for use in clinical or nonclinical decision-making and particularly within the regulatory context.

The St George's Respiratory Questionnaire (SGRQ) is a measure of health-related quality of life widely used in clinical research. The aim of the CBQC working group on SGRQ was to construct an individual patient level database of clinical trial data that included the SGRQ, to use this to confirm the reliability and validity of the SGRQ as an outcome measure of health status, and investigate its use as a predictor of future events (exacerbations and mortality). This manuscript describes the formulation of the CBQC database and presents the baseline demographic and clinical characteristics of the integrated SGRQ database overall, and by study type (short-term [ $\leq 1$ year], medium-term [2-4 years] and observational studies).

Distribution of baseline SGRQ scores varied little by demographic determinants except for income region in the observational data set (low-middle income countries +10 units compared with high income, $p<0.0001$ ) and this observation held across studies. SGRQ scores increased with increasing modified Medical Research Council dyspnea scores (mean differences ranged 6.9-17.9 units) and with increasing airflow limitations (Global initiative for chronic Obstructive Lung Disease grades 1 to 4; differences ranged 4.5-16.1 units), consistent across study types.

As a method of cross-sectional comparison, the SGRQ appears to be relatively free of bias from demographic factors although care should be taken when making cross sectional comparisons of scores between patients in countries at different levels of socio-economic development.

\footnotetext{
Abbreviations: chronic obstructive pulmonary disease, COPD; World Health Organization, WHO; health-related quality of life, HRQL; COPD Biomarkers Qualification Consortium, CBQC; National Emphysema Treatment Trial, NETT; COPD Genetic Epidemiology, COPDGene; St George's Respiratory Questionnaire, SGRQ; 6-minute Walk Test, 6MWT; forced expiratory volume in 1 second, FEV $\mathbf{1}$; body mass index, BMI; randomized controlled trial, RCT; Evaluation of COPD Longitudinally to Identify Predictive Surrogate Endpoints, ECLIPSE; Body mass index-airway Obstruction-Dyspnea-Exercise performance, BODE; modified Medical Research Council, mMRC; Global initiative for chronic Lung Disease, GOLD

Funding Support: Funding for the COPD Biomarkers Qualification Consortium working group was provided by AstraZeneca, BoehringerIngelheim, GlaxoSmithKline, Novartis and Pfizer.

Date of Acceptance: February 1, 2017

Citation: Tabberer M, Benson VS, Gelhorn H, et al. The COPD Biomarkers Qualification Consortium database: Baseline characteristics of the St George's Respiratory Questionnaire dataset. Chronic Obstr Pulm Dis. 2017;4(2):112-123. doi: https://doi.org/10.15326/ jcopdf.4.2.2017.0128
}

\section{This article has an online data supplement.}


1 Research and Development, GlaxoSmithKline, Uxbridge, United Kingdom

2 Evidera, Bethesda, Maryland

3 Research and Development, AstraZeneca, Gothenburg, Sweden

4 Boehringer Ingelheim Pharmaceuticals, Inc., Ridgefield, Connecticut

5 Division of Pulmonary, Critical Care, Sleep and Allergy, Nebraska Medical Center, Omaha

6 Research and Development, AstraZeneca, Cambridge, United Kingdom

7 Research and Development, GlaxoSmithKline, Upper Merion, Pennsylvania

8 COPD Foundation, Washington, D.C.

8 Division of Clinical Science, St George's University of London, United Kingdom

\section{Address correspondence to:}

Maggie Tabberer

Research \& Development

GlaxoSmithKline

Uxbridge, United Kingdom

Phone:+44 2089902041

Email: margaret.x.tabberer@gsk.com

\section{Keywords:}

St George's Respiratory Questionnaire; SGRQ dataset; COPD Biomarker Qualification Consortium; CBQC; baseline characteristics

\section{Introduction}

Chronic obstructive pulmonary disease (COPD) is a serious public health issue, recognized by the World Health Organization (WHO) and is currently the third leading cause of death in the United States. ${ }^{1}$ COPD impacts a patient's health status across the full range of activities of living from self-care to social and family interactions and occupation. ${ }^{2,3}$ Current therapy for COPD is focused on improving lung function and reducing inflammation, thereby reducing symptoms and improving both functionality and health-related quality of life (HRQL). ${ }^{4}$ This is currently achieved through the use of long-acting bronchodilators which are commonly used in combination with inhaled corticosteroids. The development of novel therapies for COPD may target new mechanisms of action and provide an opportunity for more personalized care. ${ }^{4}$ To fully evaluate novel mechanisms, a wider range of recognized regulatory endpoints and biomarkers are required.

The COPD Biomarkers Qualification Consortium $(\mathrm{CBQC})$ is a public-private partnership which was formed following a workshop held in January 2010 and hosted by the COPD Foundation. The workshop brought together researchers and regulators to discuss the potential for formalizing new endpoints and biomarkers for use in the development of treatments for COPD. The formation of the consortium and its aims are detailed elsewhere. ${ }^{5}$ Consortium membership includes the COPD Foundation, the National Institutes of Health, academic researchers and several pharmaceutical companies and has the aim of qualifying clinical endpoints for use in drug development. The CBQC also works with specialist research organizations to both build and verify the database and to develop and perform analyses to support these aims (Figure 1).

The goal of the CBQC is to qualify biomarkers and clinical assessment tools for specific contexts of use which define how a particular biomarker will be used in clinical or nonclinical decision-making and within the regulatory framework for the development of new medicines. Recognized contexts of use may include stratification of patient populations, use in dose ranging studies in earlier medicine development and as outcome measures in regulatory clinical trials. The initial approach of the CBQC was to source and combine large clinical datasets from the pharmaceutical industry and publicly available sources. The first working groups of the consortium were formed based on the output from the January 2010 meeting. Participating companies, AstraZeneca, Boehringer Ingelheim, GlaxoSmithKline and Novartis, have contributed patient level data from clinical trial datasets and observational studies. Publicly available data sources such as the National Emphysema Treatment Trial (NETT) and COPD Genetic Epidemiology (COPDGene) data have also been integrated into the $\mathrm{CBQC}$ database. Since not all studies include all the endpoints under consideration, each working group of $\mathrm{CBQC}$ draws only on the relevant studies for analysis.

The CBQC database therefore aggregates data from selected randomized clinical trials and observational studies to advance knowledge of clinical measures used in management of COPD patients (Table 1). Health status, measured by the St George's Respiratory Questionnaire (SGRQ), was one of the initial measures evaluated in this way, along with fibrinogen ${ }^{6,7}$ and the 6-minute walk test $(6 \mathrm{MWT}){ }^{8}$ 


\section{Figure 1. The COPD Foundation Biomarkers Qualification Consortium}

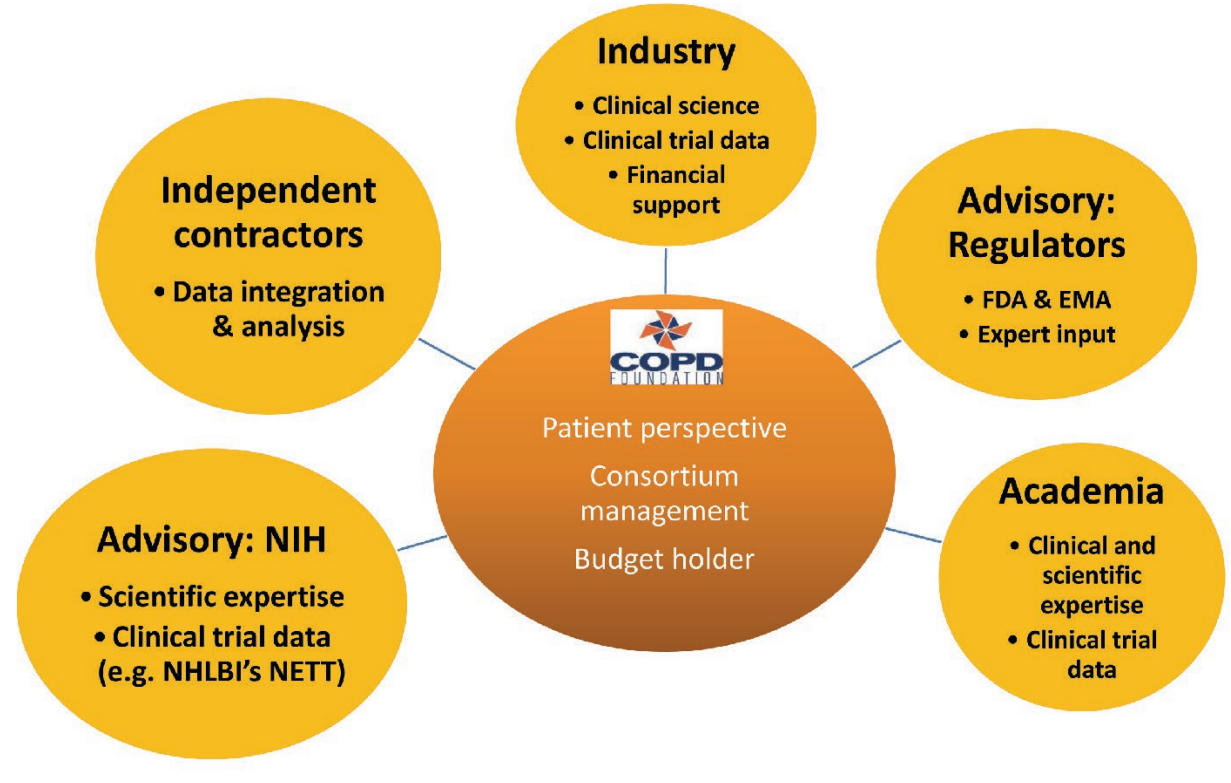

terms of cross-sectional validity and to describe longitudinal patterns and trajectories of change and predictive validity as an indicator of disease progression, morbidity, and mortality. As these analyses involved the pooling of anonymized data, National Health Service ethics approval and institutional review board approval were not required. Appropriate approvals and written informed consent had been obtained originally for all the included studies.

The objective of this manuscript is to describe the baseline demographic and clinical characteristics of the integrated SGRQ database overall, and by study type. In addition, to describe SGRQ scores within and across study types by baseline characteristics.
The SGRQ is a 50 -item patient-reported outcome measure that was designed to assess health-related quality of life in patients with chronic airflow limitation. 9,10 Specifically, the instrument was developed to evaluate the impact of airflow limitation on the patient's life in terms of symptoms, daily activity limitation, and psychosocial well-being. The SGRQ-C is a COPD specific, 40-item version of the original SGRQ and was derived following detailed analysis of data from large studies in COPD. ${ }^{11}$ The SGRQ and SGRQ-C are divided into 2 major components: Part I produces the Symptoms score, while Part 2 produces the Activity and Impacts scores; a total score is also generated. The scoring method used for both versions of the SGRQ provides equivalent $S G R Q$ scores, i.e., a score range from 0 (best health status) to 100 (worst health status).

Several clinical studies, as well as several large population-based observational studies, have included the SGRQ as a measure of health status. Although the United States Food \& Drug Administration has indicated acceptance of the SGRQ as an outcome measure for clinical trials of COPD, ${ }^{12}$ the SGRQ has until recently only been included in product labels for monotherapy bronchodilator treatments for COPD using placebo as a comparator. There remained a need for further analyses of large prospective studies to confirm the performance properties of the SGRQ total score, specifically in

\section{Methods}

Minimum data requirements were created based on an analysis plan which addressed the study objectives of the working group set out above. A total of 20 eligible studies were entered in the database for analysis, aiming to balance the geographic spread of the patients, the duration of studies and the numbers of patients included from each participating company, while maintaining the required minimum data requirements for the analysis (Figure 2). NETT, a 5-year randomized, controlled trial evaluating the effectiveness of lung volume reduction surgery, was analyzed separately due to the specialist nature of the data which could not be meaningfully combined into the SGRQ integrated dataset. Additional studies were considered, but excluded based on study design and development phase, number of patients included, and publication date (all included studies were published during or after 2003). COPD diagnosis and severity was defined by the studies included in the database. The incorporated studies covered a wide range of COPD severity, as defined by lung function and including measures of reversibility. Details of the included studies are provided in the online data supplement, Table S1.

Data were provided to a third-party database provider, INC Research, by each company for data entry (Figure 2). 


\section{Table 1. List of Studies Included in the CBQC SGRQ Database}

\section{Study \\ Key Reference}

\section{A. Short Term Clinical Trials $\leq \mathbf{1 2}$ Months in Duration}

D5899C00001: Effect of Tiotropium on Sputum and Serum

Inflammatory Markers and Exacerbations in COPD

Powrie DJ, Wilkinson TM, Donaldson GC, et al. Effect of

tiotropium on sputum and serum inflammatory markers

and exacerbations in COPD. Eur Respir J. 2007;30(3):

472-478.

D5899C00003: Effect of Budesonide/ Formoterol pMDI on COPD Exacerbations: A Double-Blind, Randomized Study

Sharafkhaneh A, Southard JG, Goldman M, Uryniak T, Martin UJ. Effect of budesonide/formoterol pMDI on COPD exacerbations: a double-blind, randomized study. Respir Med. 2012;106(2):257-268.

SD-039-0670: Maintenance Therapy with Budesonide and Formoterol in Chronic Obstructive Pulmonary Disease Calverley PM, Boonsawat W, Cseke Z, Zhong N, Peterson S, Olsson $\mathrm{H}$. Maintenance therapy with budesonide and formoterol in chronic obstructive pulmonary disease. Eur Respir J. 2003;22(6):912-919.

205.259: A Randomized Controlled Trial to Assess the Efficacy of Tiotropium in Canadian Patients with Chronic Obstructive Pulmonary Disease

Chan CK, Maltais F, Sigouin C, Haddon JM, Ford GT, Group SS. A randomized controlled trial to assess the efficacy of tiotropium in Canadian patients with chronic obstructive pulmonary disease. Can Respir J. 2007;14(8): 465-472.

205.230: Improvement in Exercise Tolerance with the Combination of Tiotropium and Pulmonary Rehabilitation in Patients with COPD

Casaburi R, Kukafka D, Cooper CB, Witek TJ, Jr., Kesten

$\mathrm{S}$. Improvement in exercise tolerance with the combination of tiotropium and pulmonary rehabilitation in patients with COPD. Chest. 2005;127(3):809-817.

SFCB3024: Combined Salmeterol and Fluticasone in the Treatment of Chronic Obstructive Pulmonary Disease: A Randomised Controlled Trial Calverley P, Pauwels R, Vestbo J, et al. Combined salmeterol and fluticasone in the treatment of chronic obstructive pulmonary disease: A randomised controlled trial. Lancet. 2003;361(9356):449-456.

207499/157: The Efficacy and Safety of Cilomilast in COPD Rennard S, Knobil K, Rabe KF, et al. The efficacy and safety of cilomilast in COPD. Drugs. 2008;68(Suppl 2):3-57.

207499/121: The Efficacy and Safety of Cilomilast in COPD Rennard S, Knobil K, Rabe KF, et al. The efficacy and safety of cilomilast in COPD. Drugs. 2008;68(Suppl 2):3-57.

QAB149B2334: Efficacy of a New Once-Daily Long-Acting Inhaled Beta2-Agonist Indacaterol Versus Twice-Daily Formoterol in COPD Dahl R, Chung KF, Buhl R, et al. Efficacy of a new oncedaily long-acting inhaled beta2-agonist indacaterol versus twice-daily formoterol in COPD. Thorax. 2010;65(6): 473-479.

QAB149b2350: Blinded 12 Week Comparison of Once Daily Indacaterol and Tiotropium in COPD

Buhl R, Dunn LJ, Disdier C, et al. Blinded 12-week comparison of once-daily indacaterol and tiotropium in COPD. Eur Respir J. 2011;38(4):797-803.

QAB149B2336: Once-Daily Indacaterol Versus Twice-Daily Salmeterol for COPD: A Placebo-Controlled Comparison Kornmann O, Dahl R, Centanni S, et al. Once-daily indacaterol versus twice-daily salmeterol for COPD: a placebo-controlled comparison. Eur Respir J. 2011;37(2): 273-279.

QAB149B2335S. Once-Daily Bronchodilators for Chronic Obstructive Pulmonary Disease Indacaterol Versus Tiotropium

Donohue JF, Fogarty C, Lotvall J, et al. Once-daily bronchodilators for chronic obstructive pulmonary disease: indacaterol versus tiotropium. Am J Respir Crit Care Med. 2010;182(2):155-162. 
205.247: Tiotropium and Exercise Training in COPD Patients: Effects on Dyspnea and Exercise Tolerance

Ambrosino N, Foglio K, Balzano G, et al. Tiotropium and exercise training in COPD patients: effects on dyspnea and exercise tolerance. Int J Chron Obstruct Pulmon Dis. 2008; 3(4):771-780.

\section{B. Medium Term Clinical Trials $>12$ Months in Duration}

Understanding Potential Long-Term Improvements in Function with Tiotropium (UPLIFT)

Decramer M, Celli B, Tashkin DP, et al. Clinical trial design considerations in assessing long-term functional impacts of tiotropium in COPD: the UPLIFT trial. COPD. 2004;1(2): 303-312.

Towards a Revolution in COPD Health (TORCH) The TORCH Study Group. The TORCH (Towards a Revolution in COPD Health) survival study protocol. Eur Respir J. 2004;24(2):206-210.

SCO40036: The Prevention of Chronic Obstructive Pulmonary Wedzicha W, Calverley PMA, Seemungal TA, Hagan G, Disease Exacerbations by Salmeterol/ Fluticasone Propionate Ansari Z, Stockley RA for the Inspire investigators. or Tiotropium Bromide Prevention of COPD exacerbations with salmeterol/ fluticasone propionate or tiotropium bromide. Am J Respir Crit Care Med. 2008;177(1):19-26.

\section{Observational Studies}

Evaluation of COPD Longitudinally to Identify Predictive Surrogate Endpoints (ECLIPSE)

Vestbo J, Anderson W, Coxson HO, et al. Evaluation of COPD longitudinally to identify predictive surrogate endpoints. Eur Respir J. 2008;31(4):869-873.

COPDGene Study Regan EA, Hokanson JE, Murphy JR, et al. Genetic Epidemiology of COPD (COPDGene) study design. COPD. 2010;7(1):32-43.

The Body Mass Index, Airflow Obstruction, Dyspnea, and Celli BR, Cote CG, Marin JM, et al. The body-mass index, Exercise (BODE) Capacity Index in Chronic Obstructive Pulmonary Disease airflow obstruction, dyspnea, and exercise capacity index in chronic obstructive pulmonary disease. $N$ Eng J Med. 2004;350(10):1005-1011.

Under the consortium agreement, all data were entered by a third party and comparisons were only made at the level of drug class during analysis. The database was provided to Evidera, a company specializing in outcomes analytics, for quality control checks and subsequent analysis. Following receipt of the data from INC Research, Evidera mapped and reconciled all variables from each study so that they might be analyzed as a single dataset. This involved review of each variable in each study in the context of how the variable was operationalized, and then manipulation of the variable so that studies could subsequently be analyzed together in the full database. In addition, some variables were derived upon receipt of the database (i.e., study drop-out status), as they were relevant for analysis but were not calculated in the data files transferred.

This quality control process was designed to ensure the accuracy of the data for subsequent analyses. An iterative process was developed and individually applied to each study dataset. Baseline descriptive demographic and clinical characteristics were tabulated, as well as available longitudinal measures (SGRQ, 6MWT, forced expiratory volume in $1 \mathrm{sec}\left[\mathrm{FEV}_{1}\right]$ ), and medical-related events tables from each individual study. Logic checks were then conducted to ensure that these variables were within a feasible predefined range, (e.g., SGRQ scores were all between 0 and 100), and that the values appeared reasonable based on the study design and the disease severity of the enrolled COPD population. These descriptive tables were then compared to a primary publication for each study. Where discrepancies were found, individual study sponsors were contacted to reconcile identified issues or discrepancies. Following this step each study sponsor completed and signed a review form documenting their review and any discrepancies they may have identified, and then emailed this form to Evidera. Any outstanding issues were then resolved with assistance from the sponsor, the data integration company (INC Research), or the SGRQ working group, as necessary. 
Figure 2. Disposition of the Short-Term, MediumTerm, and Observational Datasets

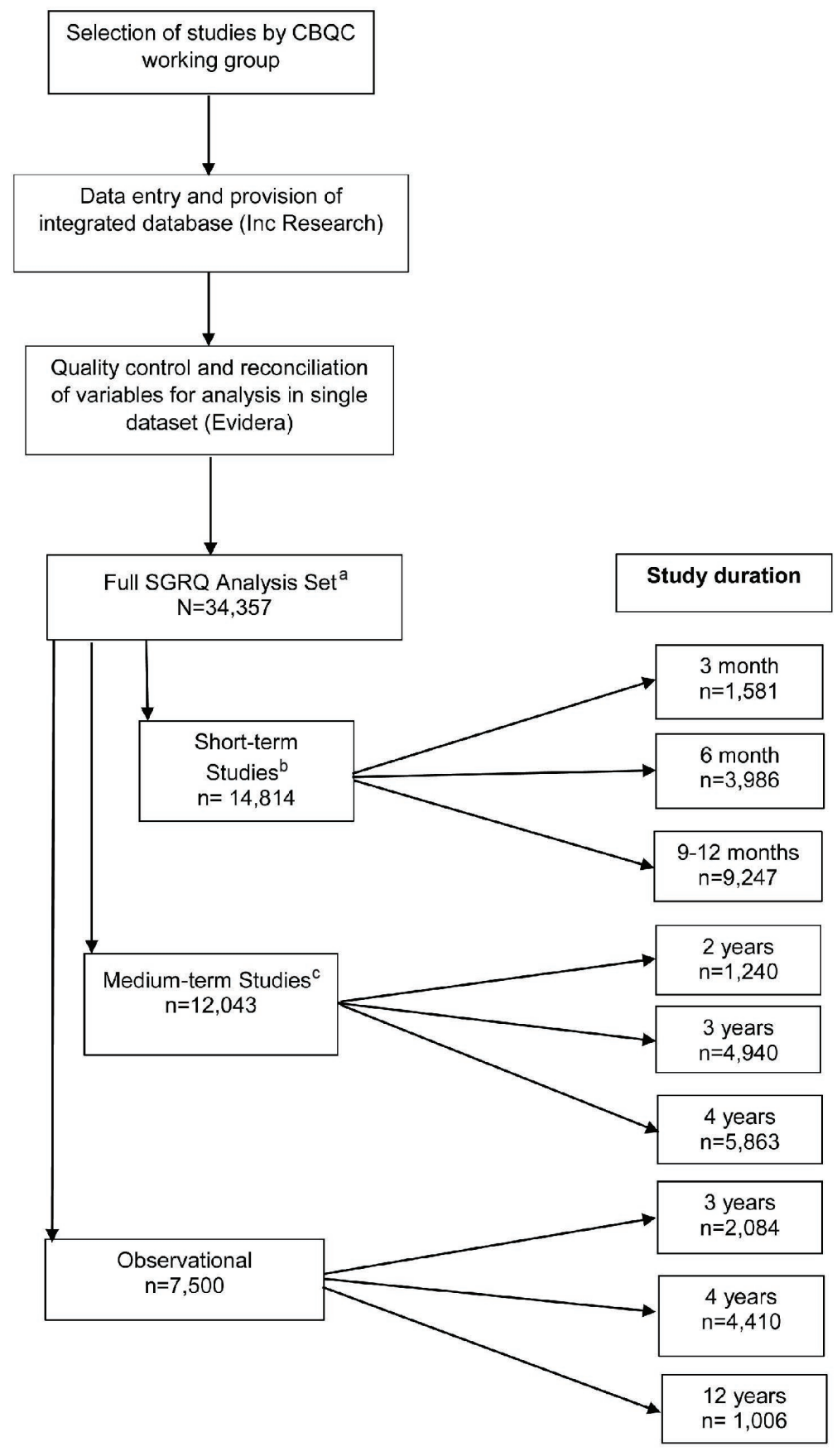

${ }^{a}$ To be included in the dataset, individuals had to have an SGRQ score at baseline and fall in
an eligible study arm; ${ }^{b}$ Short-term studies: $\leq 12$ months; ${ }^{c}$ Medium term studies: $>12$ months

following as covariates: gender, age, race (white/non-white), categorical body mass index (BMI), WHO classification of region and income, study type (short-term, medium-term, observational), year study began, and smoking status (current/past).

\section{Results}

A total of 20 studies were available for analysis in the SGRQ aggregated database: 17 randomized controlled trials (RCTs) and 3 observational studies (ECLIPSE [Evaluation of COPD Longitudinally to Identify Predictive Surrogate Endpoints], ${ }^{13}$ n=2084; COPDGene, ${ }^{14}$ n=4410; and Body mass index-airway Obstruction-DyspneaExercise performance [BODE], $\left.{ }^{15} \mathrm{n}=1006\right)$ (Figure 2). The 17 RCTs were grouped by study duration, categorized as short-term ( $\leq 1$-year duration), comprising 14 trials with 14,814 participants, and mediumterm (2-4 years' duration) comprising 3 trials with 12,043 participants. Further details of each included study are provided in the online data supplement Table S1.

The mean age in the integrated database was 64.1, and age was similar across all study types (Table 2). Mean BMI in the integrated dataset was $26.3 \mathrm{~kg} / \mathrm{m}^{2}$, and was also fairly consistent across the 3 study types. Observational studies had more females (37\%) than either of the short- $(29 \%)$ or medium-term (24\%) clinical trial datasets, and mean $\mathrm{FEV}_{1} \%$ predicted was higher in observational studies (53.3\%) compared with the short- (47.2\%) and medium-term trials (45.0\%). Dyspnea, measured at baseline by modified Medical Research Council dyspnea grade (mMRC) and defined as a score $\geq 2$, was reported by $63.6 \%$ of patients in the integrated dataset, with higher rates in the short-term trials (69.7\%), relative to both the medium-term trials (59.9\%) and observational (59.6\%)

Multivariate regression models were conducted to evaluate relationships between baseline SGRQ and demographic and clinical characteristics, with baseline SGRQ total score as the dependent variable, and the study populations. Data on history of cardiovascular disease (including hypertension) were missing for over $80 \%$ of patients in short-term trials, whereas the majority of these data were available for medium-term trials and 


\section{Table 2. Demographic and Clinical Characteristics of COPD Patients}

\begin{tabular}{|c|c|c|c|c|c|}
\hline & & $\begin{array}{c}\text { Short- } \\
\text { term Trials }\end{array}$ & $\begin{array}{l}\text { Medium- } \\
\text { term Trials }\end{array}$ & $\begin{array}{l}\text { Observational } \\
\text { Studies }\end{array}$ & $\begin{array}{l}\text { Integrated } \\
\text { Database }\end{array}$ \\
\hline \multirow[t]{2}{*}{ SGRQ Score } & $\mathrm{N}$ & 14,814 & 12,043 & 7500 & 34,357 \\
\hline & Mean (SD) & $47.3(18.3)$ & $47.8(17.2)$ & $41.8(22.2)$ & $46.3(19.0)$ \\
\hline \multirow[t]{2}{*}{ Age (years) } & $\mathrm{N}$ & 14,814 & 12,043 & 7500 & 34,357 \\
\hline & Mean (SD) & $63.8(8.9)$ & $64.7(8.4)$ & $63.6(8.4)$ & $64.1(8.6)$ \\
\hline \multirow[t]{2}{*}{ Sex (female) } & $\mathrm{N}$ & 14,814 & 12,043 & 7500 & 34,357 \\
\hline & $\mathrm{n}(\%)$ & $4236(28.6)$ & $2937(24.4)$ & $2792(37.2)$ & $9965(29.0)$ \\
\hline \multirow[t]{2}{*}{ Low-middle Income (WHO status) } & $\mathrm{N}$ & 14,814 & 12,043 & 7500 & 34,357 \\
\hline & $\mathrm{n}(\%)$ & $5252(35.5)$ & $3732(31.0)$ & $292(6.5)$ & $9276(27.0)$ \\
\hline \multirow[t]{2}{*}{ FEV $_{1} \%$ Predicted } & $\mathrm{N}$ & 14,785 & 12,034 & 7494 & 32,513 \\
\hline & Mean (SD) & $47.2(14.8)$ & $45.0(12.7)$ & $53.3(21.1)$ & $47.8(16.0)$ \\
\hline \multicolumn{6}{|l|}{ FEV $_{1} \%$ Predicted Categories } \\
\hline Mild (> 80\%) & $\mathrm{n}(\%)$ & $144(1.0)$ & $7(0.06)$ & $829(11.1)$ & $980(2.9)$ \\
\hline Moderate $(>50 \%$ and $<80 \%)$ & $\mathrm{n}(\%)$ & 5859 (39.6) & 4607 (38.3) & $3129(41.8)$ & $13,595(39.6)$ \\
\hline Severe $(>30 \%$ and $<50 \%)$ & n (\%) & 7015 (47.4) & $5848(48.6)$ & $2457(32.8)$ & $15,320(44.6)$ \\
\hline Very Severe $(\leq 30 \%)$ & $\mathrm{n}(\%)$ & $1767(12.0)$ & $1572(13.1)$ & $1079(14.4)$ & $4418(12.9)$ \\
\hline \multirow[t]{2}{*}{ Current Smoker } & $\mathrm{N}$ & 14,807 & 12,043 & 7493 & 34,343 \\
\hline & n (\%) & $5907(39.9)$ & $4446(36.9)$ & $2971(39.7)$ & $13,324(38.8)$ \\
\hline \multirow[t]{2}{*}{ Body Mass Index $\left(\mathrm{kg} / \mathrm{m}^{2}\right)$} & $\mathrm{N}$ & 14,804 & 12,043 & 7500 & 34,347 \\
\hline & Mean (SD) & $26.09(5.33)$ & $25.78(5.12)$ & $27.4(6.0)$ & $26.3(5.4)$ \\
\hline \multirow[t]{2}{*}{ mMRC $\geq 2$} & $\mathrm{~N}$ & 8591 & 6176 & 7430 & 22,197 \\
\hline & $\mathrm{n}(\%)$ & $5989(69.7)$ & $3698(59.9)$ & 4427 (59.6) & $14,114(63.6)$ \\
\hline \multirow{2}{*}{$\begin{array}{l}\text { History of Cardiovascular Disease, } \\
\text { Including Hypertension }\end{array}$} & $\mathrm{N}$ & 2311 & 12,042 & 7360 & 21,713 \\
\hline & $\mathrm{n}$ (\% with CVD) & $1181(51.1)$ & $3224(26.8)$ & $2096(28.5)$ & $6501(29.9)$ \\
\hline \multirow{2}{*}{$\begin{array}{l}\text { Number of Exacerbations in Previous } \\
12 \text { Months }\end{array}$} & $\mathrm{N}$ & 8891 & 6180 & 6494 & 21,565 \\
\hline & Mean (SD) & $0.64(1.07)$ & $1.59(2.50)$ & $0.73(1.21) \dagger$ & $0.94(1.70)$ \\
\hline
\end{tabular}

SGRQ=St George's Respiratory Questionnaire; WHO=World Health Organization; FEV 1 =forced expiratory volume in 1 second; $\mathrm{mMRC}=$ modified Medical Research Council dyspnea scale; $\mathrm{SD}=$ standard deviation; $\mathrm{CVD}=$ cardiovascular disease

observational studies, yet the percentage of patients reported as experiencing a cardiovascular event was very similar for these 2 populations (27\% and 29\%, respectively).

The mean baseline SGRQ score in the integrated database was 46.3, and scores were higher (worse health status) in both clinical trial populations (short-term $=$ 47.3, medium-term $=47.8$ ) relative to the observational study population (41.8) (Table 2). The integrated database included a wide geographical distribution of patients, with more patients from low-middle income countries in both the short- (35.5\%) and medium-term (31.0\%) trials compared with the observational studies (6.5\%).

As expected the mean number of exacerbations reported in the 12 months prior to study initiation varied considerably by study type as this variable is driven by study design and inclusion/exclusion criteria.
Exacerbation data, collected either as an endpoint or as adverse event data were available only in $60 \%$ of shortterm trials, $51.3 \%$ of medium-term trials, and $86.6 \%$ of the observational population. Among patients that had exacerbation data available, annual exacerbation rates ranged from 0.64 (short-term trials) to 1.59 (mediumterm trials).

Across the 3 study types, distribution of baseline SGRQ score varied little by demographic determinants (Figure 3). For the demographic determinants analyzed, WHO income region was the only factor where a difference in SGRQ score between groups at baseline exceeded the 4-unit minimum clinically important difference, observed in the observational population only (low-middle income countries +10 units compared to high income), $p<0.0001$. Small and non-significant differences were seen in the baseline SGRQ scores based on smoking status, gender and age, dependent on study 


\section{Figure 3. Demographic Determinants of SGRQ Score}

\section{A. Short-term studies}

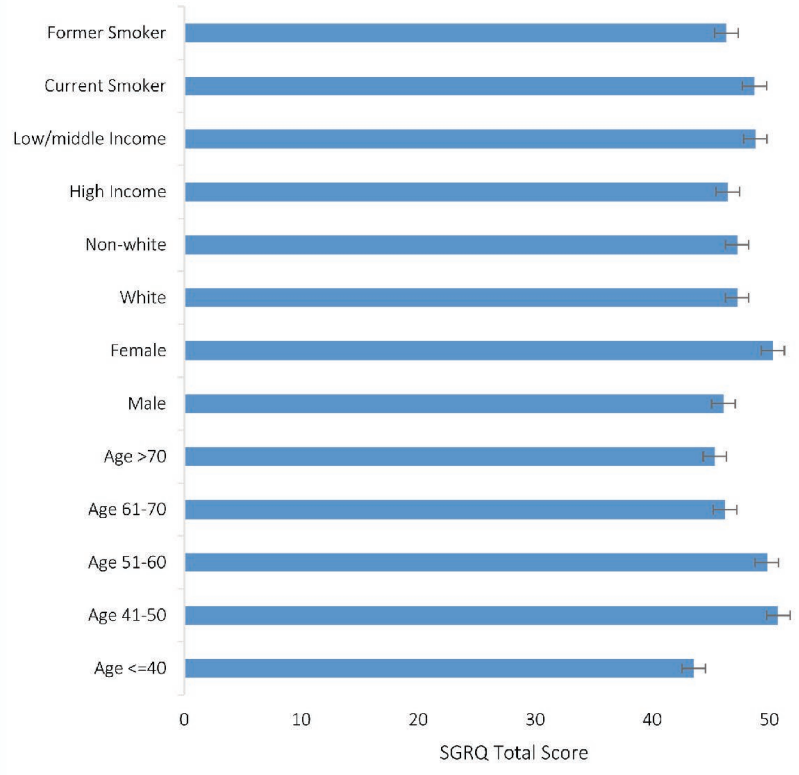

\section{B. Medium-term studies}

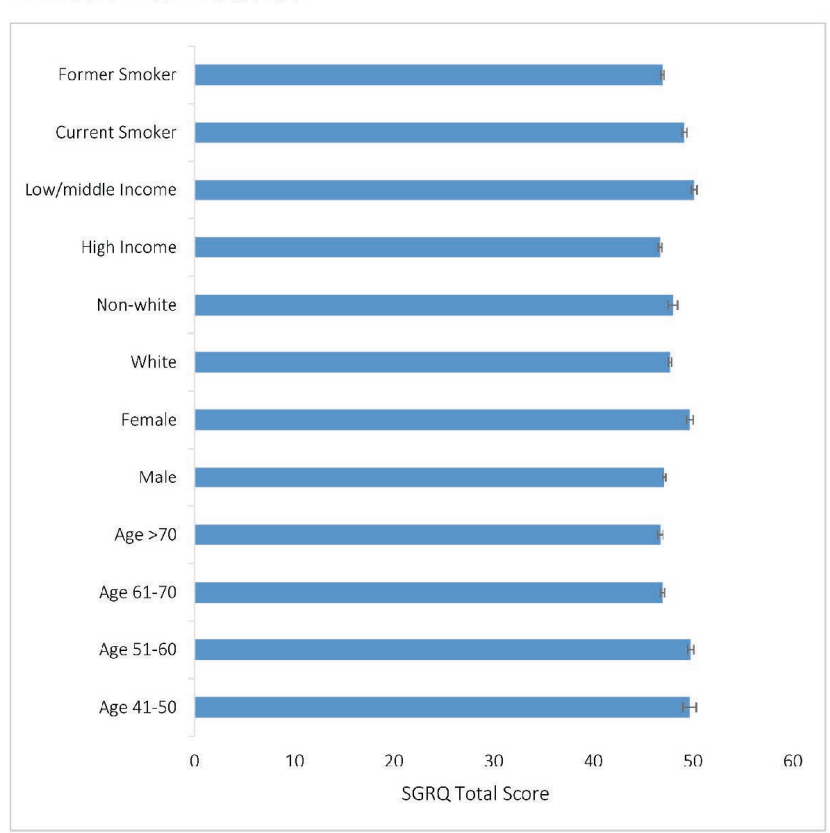

\section{Observational studies}

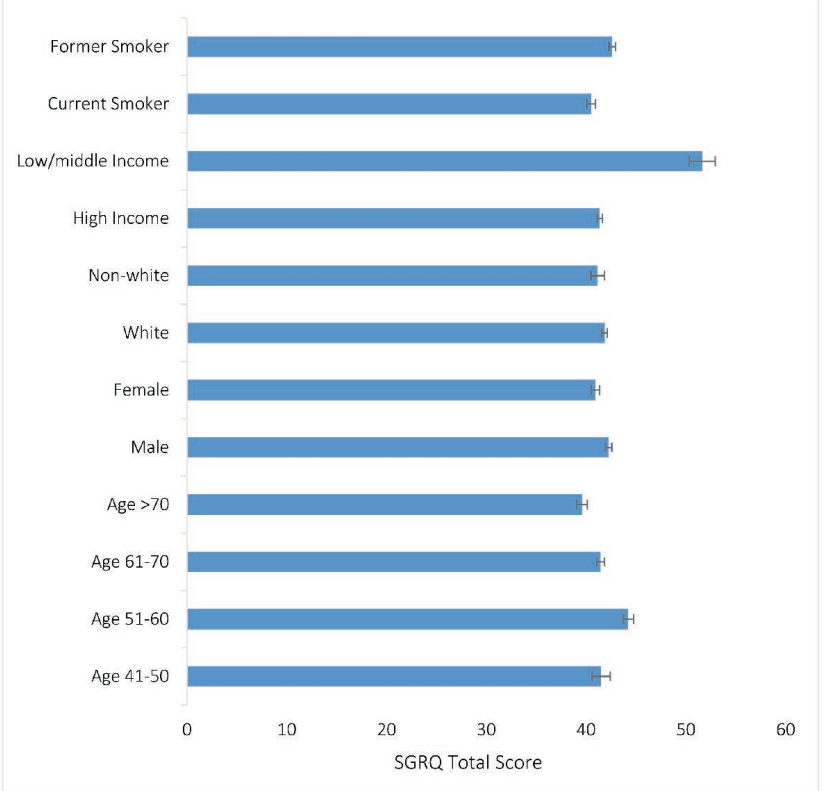

Data are mean $\pm \mathrm{SE}$

population and design. Although current smokers, women and younger patients (aged 41-60 years) reported slightly higher baseline SGRQ scores than exsmokers, men and older patients, these differences were not significant and were not consistent across all study types.

Disease-related determinants were related to baseline SGRQ score with scores increased in line with increasing $\mathrm{mMRC}$ dyspnea score and with declining lung function when defined by Global initiative for chronic Obstructive Lung Disease (GOLD) ${ }^{4}$ grade of airflow limitation (1 to 4), and this was consistent across study types (Figure 4). These differences ranged from a mean of 6.9-17.9 units for mMRC dyspnea score and a mean of 4.5-16.1 units for GOLD grade. The presence of comorbid cardiovascular disease was also associated 


\section{Figure 4. Disease-related Determinants of SGRQ Score}

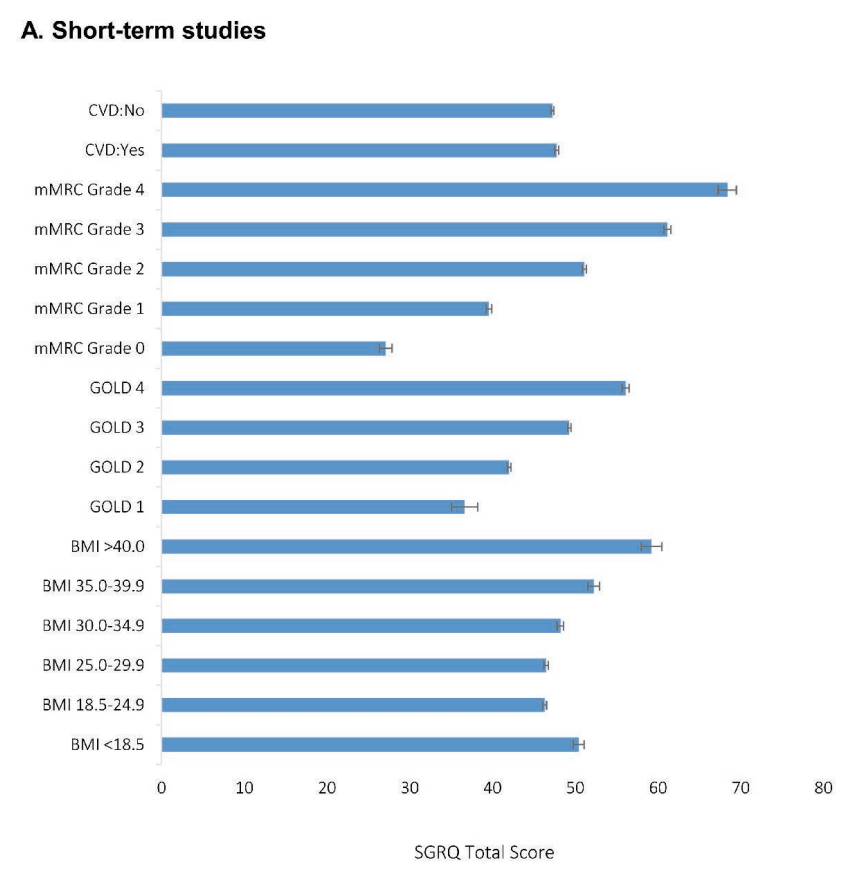

B. Medium-term studies

\section{Observational studies}
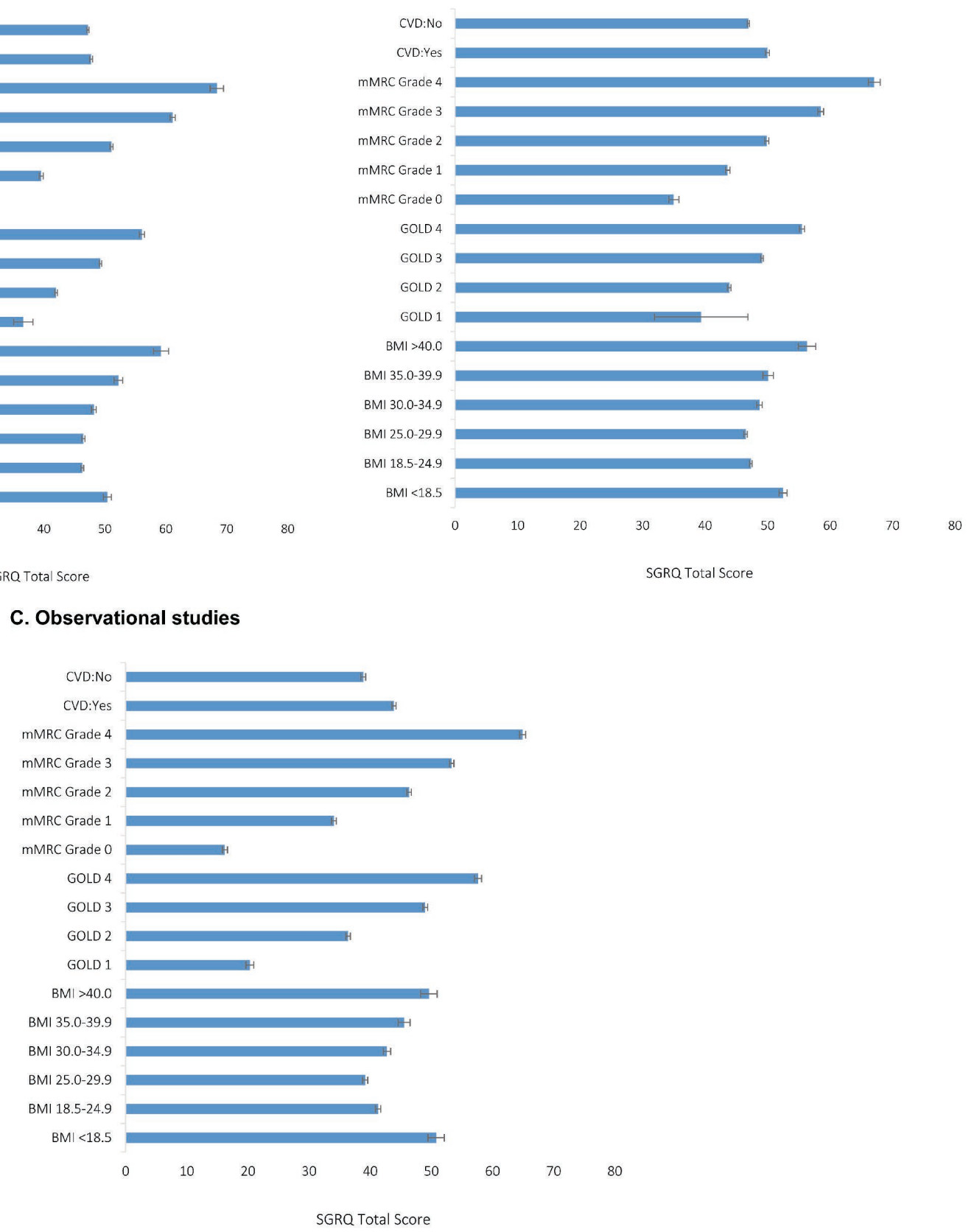

Data are mean and $\pm \mathrm{SE}$

with a +5-point higher baseline SGRQ score in the observational population, compared with +3.1 and +0.6 in the medium-term and short-term trials respectively. BMI showed a U-shaped relationship with SGRQ score across all study types, with higher SGRQ scores at the extremes of the reported BMI range.

Baseline SGRQ score and $\mathrm{FEV}_{1}$ percent predicted were poorly correlated in the interventional studies
(Short-term: $\mathrm{r}=-0.28$; Medium-term: $\mathrm{r}=-0.26$ ); a stronger relationship was seen in the Observational data set ( $\mathrm{r}=$ -0.53) (Figure 5). Longitudinally, for the integrated dataset, the correlation between change in SGRQ and FEV 1 percent predicted change, $r$ ranged from 0.100.27 across treatment classes (placebo, long-acting bronchodilators and inhaled corticosteroid/long-acting beta2-agonist) and follow up time between 3 and 36 


\section{Figure 5. Relationship Between SGRQ and FEV ${ }_{1} \%$ Predicted}

\section{A. Short-term studies}

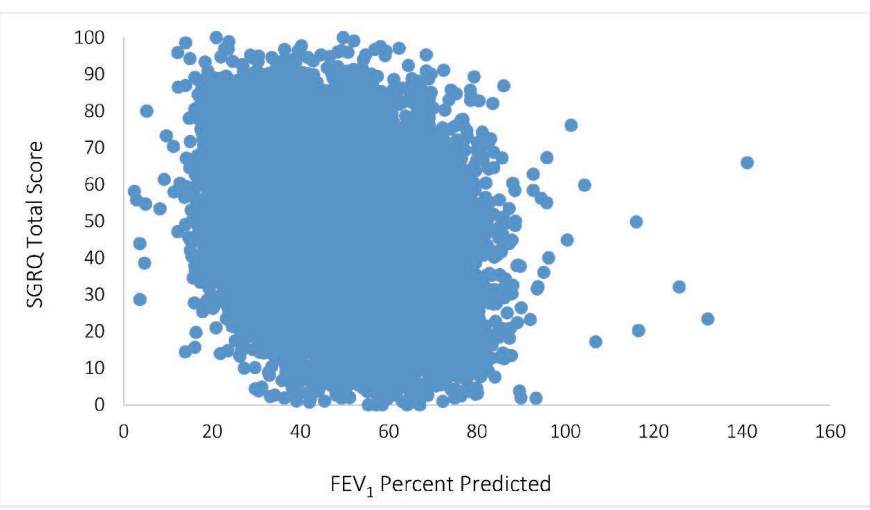

B. Medium-term studies

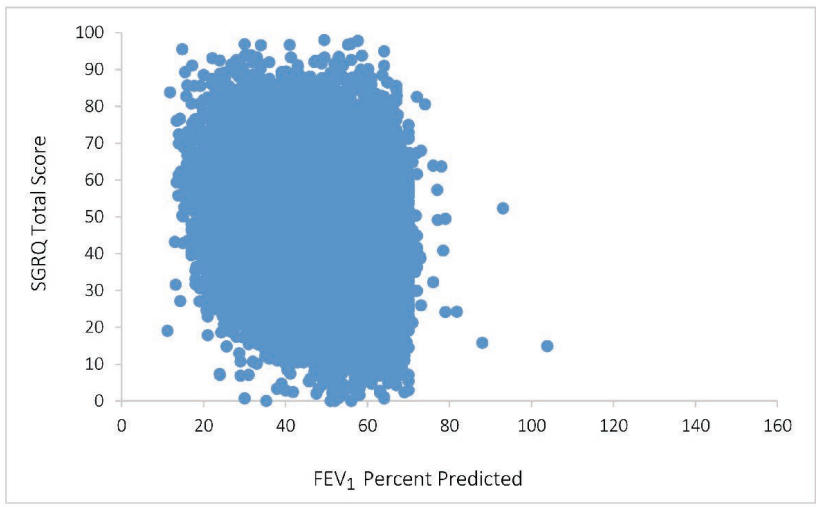

C. Observational studies

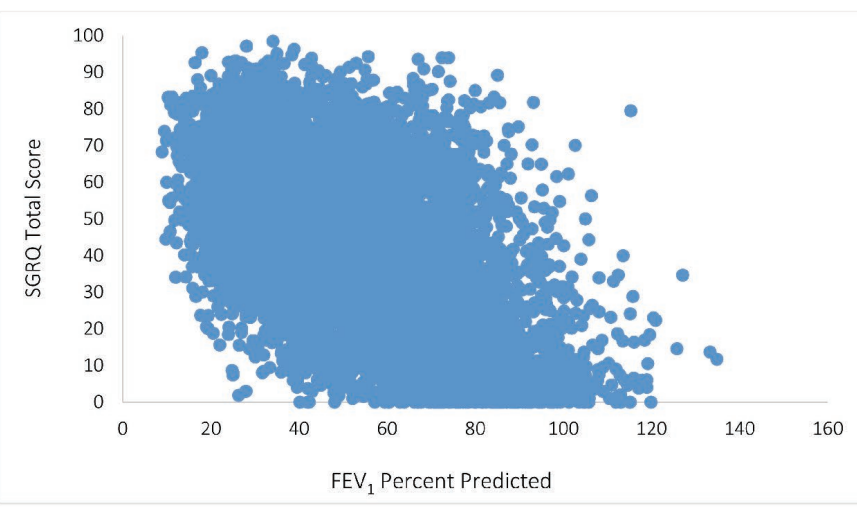

months. The correlation between change in SGRQ and transition dyspnea index over a similar time period (up to 24 months) and for the same treatments (but not identical studies) was 0.31-0.47.

\section{Discussion}

The combination of RCTs and observational studies in the CBQC database enables analysis of baseline SGRQ scores over a wide variety of demographic and diseaserelated determinants by providing access to data from patients with a range of disease severity. As expected, baseline SGRQ scores varied by mMRC score, and GOLD grade. There were differences between different demographic groups, but these were generally small (less than the 4-unit minimum clinically important difference for the SGRQ) and not consistent between studies. For example, in the RCTs, smokers had higher scores than former smokers and women were higher than men, but these differences were not seen in the observational studies. There was no consistent effect of age across the different types of study either.
The variation in score attributable to socio-economic status seen in the observational studies is noteworthy since it was large. Specifically, patients in low-medium income countries had mean scores that were high $(>50$ units) and on average $>10$ units higher than those in high income countries, but this differed from the picture seen in the clinical trials, both short- and medium-term. The reason for this is not clear, but may be due to an effect of standardized inclusion criteria for patients recruited to trials that could have minimized the inclusion of patients with very poor health status. When considering the data from the observational studies in this context, it is important to appreciate that only 1 low-medium income country (Venezuela) contributed patients, this was in the BODE cohort. ${ }^{15}$ All other patients in BODE and the other 2 observational studies came from high income countries. While it is possible that the difference seen in Venezuela was due to language, another cohort study from 6 western-European countries, that included Spain, showed quite small differences in mean SGRQ scores between them. ${ }^{16}$ It is not possible to draw any firm conclusions about income and SGRQ score based 
on data from 1 country, and the absence of a similar pattern in the RCTs suggests that income may not be an important determinant of disease-specific health status score. Neither cardiovascular comorbidity nor baseline BMI measurement had a large impact on baseline SGRQ score, so overall it appears that demographic influences on SGRQ score are small, which is important when translating the results of studies into clinical practice and for more naturalistic or observational study designs which may recruit from a wider pool of patients. The poor correlation between baseline SGRQ score and FEV 1 percent predicted seen in the RCTs was expected and has been reported often before, however the stronger correlation in the observational studies is notable and is probably due to the much wider range of $\mathrm{FEV}_{1}$ values in the participants in these studies, a clear proportion of whom had $\mathrm{FEV}_{1}$ values above $80 \%$ predicted (i.e., in the normal range). There are few reported data in individuals with this degree of airflow limitation (or none) so this observation is relatively novel.

\section{Conclusions}

As a method of cross-sectional comparison, the SGRQ appears to be relatively free of bias from demographic factors. Care should be taken in cross-sectional comparison of scores between patients in countries at different levels of socio-economic development.

\section{Acknowledgements}

The authors would like to thank Debbie Merrill, COPD Foundation, for managing the review process, and the CBQC for their role in aggregating the data. They would also like to thank Tom Martin of Novartis and Katja Rüdell, formerly of Pfizer, for their review and oversight through the CBQC Steering Committee. They also acknowledge the assistance provided by Kate Hollingworth of Continuous Improvement Ltd in copyediting and formatting the manuscript; this was funded by the COPD Foundation.

\section{Declaration of Interest}

MT, VSB, NK, HM, SM, SIR, RTS, and PWJ are employees of the pharma companies who funded this analysis. SIR is also an employee of the University of Nebraska Medical Center. HG and HW are employees of Evidera, a company which performs work for hire for multiple pharmaceutical and device companies in outcomes research, and were funded by the CBQC consortium. DM has nothing to declare. 


\section{References}

1. Kochanek KD, Murphy SL, Xu J, Tejada-Vera B. Deaths: Final data for 2014. Nat Vital Stat Rep. 2016;65(4).

2. Lopez AD, Shibuya K, Rao C, et al. Chronic obstructive pulmonary disease: current burden and future projections. Eur Respir J. 2006;27(2):397-412.

doi: https://doi.org/10.1183/09031936.06.00025805

3. Mathers CD, Loncar D. Projections of global mortality and burden of disease from 2002 to 2030. PLoS Med. 2006;3:e442. doi: https://doi.org/10.1371/journal.pmed.0030442

4. Global Initiative for Chronic Lung Disease (GOLD). Global Strategy for the Diagnosis, Management and Prevention of COPD. GOLD website. http://goldcopd.org/gold-2017-globalstrategy-diagnosis-management-prevention-copd/ Published 2017. Accessed January 2017.

5. Casaburi R, Celli B, Crapo J, et al. The COPD Biomarker Qualification Consortium (CBQC). COPD. 2013;10(3):367-377. doi: https://doi.org/10.3109/15412555.2012.752807

6. Miller BE, Tal-Singer R, Rennard SI. Plasma fibrinogen qualification as a drug development tool in chronic obstructive pulmonary disease. Perspective of the chronic obstructive pulmonary disease biomarker qualification consortium. Am J Respir Crit Care Med. 2016;193(6):607-613.

doi: https://doi.org/10.1164/rccm.201509-1722PP

7. Mannino DM, Tal-Singer R, Lomas DA, et al. Plasma fibrinogen as a biomarker for mortality and hospitalized exacerbations in people with COPD. Chronic Obstr Pulm Dis. 2015;2(1): 23-34. doi: https://doi.org/10.15326/jcopdf.2.1.2014.0138

8. Celli B, Tetzlaff $\mathrm{K}$, Criner $\mathrm{G}$, et al on behalf of COPD Biomarker Qualification Consortium. The 6-minute walk test as a COPD stratification tool: Insights from the COPD Biomarker Qualification Consortium. Am J Respir Crit Care Med. 2016. 194(12). doi: https://doi.org/10.1164/rccm.201508-1653OC

9. Jones PW, Quirk FH, Baveystock CM. The St George's Respiratory Questionnaire. Respir Med. 1991;85(Suppl 2):25-31. doi: https://doi.org/10.1016/S0954-6111(06)80166-6

10. Jones PW, Quirk FH, Baveystock CM, Littlejohns P. A selfcomplete measure of health status for chronic airflow limitation: The St. George's Respiratory Questionnaire. Am Rev Respir Dis. 1992; 145(6):1321-1327.

doi: https://doi.org/10.1164/ajrccm/145.6.1321

11. Meguro M, Barley EA, Spencer S, Jones PW. Development and validation of an improved, COPD-specific version of the St. George's Respiratory Questionnaire. Chest. 2007; 132(2): 456463. doi: https://doi.org/10.1378/chest.06-0702
12. Food and Drug Administration (FDA). Draft guidance. Chronic obstructive pulmonary disease: Developing drugs for treatment guidance for industry. FDA website. http://www.fda.gov/ downloads/drugs/guidancecomplianceregulatoryinformation/ guidances/ucm071575.pdf Published May 2016. Accessed January 2017.

13. Vestbo J, Anderson W, Coxson HO, et al. Evaluation of COPD Longitudinally to Identify Predictive Surrogate End-points (ECLIPSE). Eur Respr J. 2008; 31(4): 869-873. doi: https://doi.org/10.1183/09031936.00111707

14. Regan EH, Hokanson JE, Murphy JR, et al. Genetic epidemiology of COPD (COPDGene) study design. COPD. 2010;7(1):32-43. doi: https://doi.org/10.3109/15412550903499522

15. Celli BR, Cote CG, Marin JM, et al. The body-mass index, airflow obstruction, dyspnea, and exercise capacity index in chronic obstructive pulmonary disease. N Eng J Med. 2004;350(10):10051011. doi: https://doi.org/10.1056/NEJMoa021322

16. Jones PW, Brusselle G, Dal Negro RW, et al. Health-related quality of life in patients by COPD severity within primary care in Europe. Respir Med. 2011;105(1):57-66. doi: https://doi.org/10.1016/j.rmed.2010.09.004 